TITLE:

\title{
Effects of internal electric field and carrier density on transient absorption spectra in a thin GaN epilayer
}

\section{AUTHOR(S):}

Omae, K; Kawakami, Y; Fujita, S; Yamada, M; Narukawa, Y; Mukai, T

\section{CITATION:}

Omae, $\mathrm{K}$... [et al]. Effects of internal electric field and carrier density on transient absorption spectra in a thin GaN epilayer. PHYSICAL REVIEW B 2002, 65(7): 073308.

\section{ISSUE DATE:}

2002-02-15

URL:

http://hdl.handle.net/2433/50158

RIGHT:

Copyright 2002 American Physical Society 
PHYSICAL REVIEW B, VOLUME 65, 073308

\title{
Effects of internal electric field and carrier density on transient absorption spectra in a thin GaN epilayer
}

\author{
Kunimichi Omae, Yoichi Kawakami, and Shigeo Fujita \\ Department of Electronic Science and Engineering, Kyoto University, Kyoto 606-8501, Japan
}

Motokazu Yamada, Yukio Narukawa, and Takashi Mukai

Nitride Semiconductor Research Laboratory, Nichia Corporation, 491 Oka Kaminaka, Anan, Tokushima 774-8601, Japan

(Received 10 September 2001; revised manuscript received 8 November 2001; published 1 February 2002)

\begin{abstract}
Carrier dynamics in a thin GaN epilayer with a thickness of $0.15 \mu \mathrm{m}$ almost coherently grown on $\mathrm{Al}_{0.2} \mathrm{Ga}_{0.8} \mathrm{~N}$ was investigated using transient absorption spectroscopy above and below the band gap. The absorption peak of excitons was observed at $3.521 \mathrm{eV}$ without a pump beam. Just after the photoexcitation above the band gap, the bleaching occurred and the absorption peak of excitons disappeared due to highdensity photogenerated carriers. At about $30 \mathrm{ps}$, the absorption peak was restored at $3.527 \mathrm{eV}$, as the carriers were decreased owing to radiative and/or nonradiative recombination. After that, the absorption spectra were larger than that without a pump beam. This is because excitonic absorption suppressed by both Franz-Keldysh and Stark effects is restored by photoinduced carriers as a result of the screening of the internal electric field.
\end{abstract}

DOI: 10.1103/PhysRevB.65.073308

PACS number(s): 78.20.-e, 71.35.-y, 78.47.+p, 77.65.Ly

GaN based semiconductors have recently attracted a lot of attention for their application as light-emitting diodes (LED's) (Refs. 1,2) and laser diodes (LD's). ${ }^{3-5}$ Some groups claim that large Stokes-like shifts are just due to an internal electric field, ${ }^{6-8}$ but others report that the localization caused by potential fluctuations plays an important role for the radiative recombination. ${ }^{9-12}$ However, it is difficult with conventional spectroscopy to separate two mechanisms by assessing InGaN epilayers, which is usually used for active layers of LED's and LD's. A transient absorption spectroscopy is a powerful tool for investigating not only carrier dynamics but also the absorption spectra depending on the carrier density. This is because the carrier density is tunable in the same sample by adjusting the optical delay between pump and probe beams. Although there have been a few reports on femtosecond transient absorption spectroscopy in GaN epilayers, ${ }^{13-15}$ they are mainly related to the ultrafast carrier dynamics related to the energy transfer to the bottom of the band. Photoinduced absorption in GaN/AlGaN single quantum well (SQW) due to internal and built-in electric fields has been reported by employing degenerate pump-andprobe $(\mathrm{P} \& \mathrm{P})$ experiments at room temperature. ${ }^{16}$ However, it was rather difficult to assess the detailed spectroscopic modulation using single laser source for both pump and probe beams, and to distinguish various factors, such as screening of internal electric fields, band filling of localized centers generated by potential fluctuation of quantum confinement, and band gap renormalization induced by many body effects. In this paper, we investigate transient absorption spectra by $\mathrm{P} \& \mathrm{P}$ spectroscopy using white light as probe beam in a $0.15-\mu \mathrm{m}$-thick undoped $\mathrm{GaN}$ epilayer on $\mathrm{Al}_{0.2} \mathrm{Ga}_{0.8} \mathrm{~N}$ at $10 \mathrm{~K}$, paying attention to the effects of internal electric fields and photoinduced carriers on the modulation of excitonic absorption.

The sample used in this study was grown by the two-flow metal organic chemical vapor deposition (MOCVD) method. The structure consists of an undoped $1 \mu \mathrm{m} \mathrm{Al}_{0.2} \mathrm{Ga}_{0.8} \mathrm{~N}$ layer grown on $c$-plane sapphire followed by an undoped $0.15 \mu \mathrm{m} \mathrm{GaN}$ layer.
The transient absorption spectroscopy depicted was performed for the measurement of temporal behavior of absorption using a dual photodiode array in conjunction with a 25 $\mathrm{cm}$ monochromator. The pump beam (340 nm:3.646 eV and $360 \mathrm{~nm}: 3.443 \mathrm{eV}$ above and below band gap, respectively) was created using an optical parametric amplifier which was pumped by the output beam from a $1 \mathrm{kHz}$ regenerative amplifier. The white light used for the probe beam was generated by focusing a part of the output beam from the regenerative amplifier on a $\mathrm{D}_{2} \mathrm{O}$ cell. The pulse width of both the pump and probe beam was $150 \mathrm{fs}$. Optical delay time of the probe beam with respect to the pump beam was tuned by changing the position of the retroreflector which could be controlled by the pulse stage. In order to detect the probe beam with spatially uniform carrier distribution in the sample, the focus size of the pump beam (300 $\mu \mathrm{m}$ in diameter) was set so as to be much larger than that of the probe beam (100 $\mu \mathrm{m}$ in diameter). Furthermore, the probe beam was perpendicularly polarized with respect to the pump beam, and the transmitted probe beam polarized in this direction was detected to avoid the scattered component of the pump beam. All of the measurements were performed at $10 \mathrm{~K}$.

Figure 1 shows the $\mathrm{cw}$ photoluminescence (PL) spectrum under a He-Cd laser excitation, as well as the reflection and the absorption spectra. The PL peak is located at $3.504 \mathrm{eV}$ and the PL linewidth is about $22 \mathrm{meV}$. The PL linewidth is much broader than that (a few meV) of a typical GaN epilayer with a few micron thickness directly grown on sapphire substrate via low temperature grown GaN buffer layer. The absorption peak of excitons is observed at $3.521 \mathrm{eV}$. Free excitons in hexagonal-GaN $(h-\mathrm{GaN})$ are composed of three bands labeled $E_{X A}, E_{X B}$, and $E_{X C}$, that are transitions from the conduction $\left(\Gamma_{7 c}\right)$ to the $\mathrm{A}\left(\Gamma_{9 v}\right), \mathrm{B}\left(\Gamma_{7 u v}\right)$, and $\mathrm{C}\left(\Gamma_{7 l v}\right)$ valence bands, respectively. Among them, $E_{X A}$ and $E_{X B}$ bands are much pronounced than the $E_{X C}$ band considering their oscillator strength. However, it is not possible to ob- 


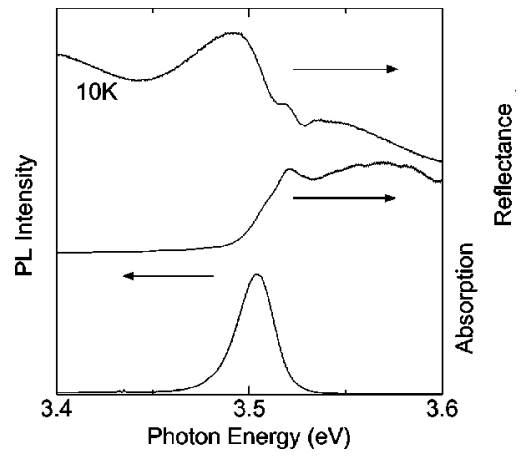

FIG. 1. PL spectrum of a thin GaN epilayer at $10 \mathrm{~K}$ under $\mathrm{cw}$ excitation with a He-Cd laser $(325 \mathrm{~nm})$. Absorption and reflectance spectra are also inserted in the figure. The absorption peak of excitons is located at $3.521 \mathrm{eV}$.

serve two absorption peaks probably because of the broadness of each component. Nevertheless, the shoulder structure appeared at around $3.510 \mathrm{eV}$. The absorption peak at 3.521 $\mathrm{eV}$ is shifted towards higher photon energy side by about 43 meV compared to the transition of $E_{X A}$ in the nearly strainfree homoepitaxial $h$-GaN layer. ${ }^{17}$ From the x-ray diffraction, the lattice constant $c$ has a large value of $5.194 \AA$ compared to $5.185 \AA$ of the strain-free $\mathrm{GaN} .{ }^{18} \mathrm{~A} \mathrm{GaN}$ epilayer suffers a large amount of in-plane biaxial compressive strain. This is caused by the thermal expansion coefficient and the lattice constant differences in the heterostructures GaN/AlGaN and AlGaN/sapphire. From Refs. 19,20, the energy positions of $E_{X A}$ and $E_{X B}$ as a function of the strain are expressed as follows, respectively,

$$
\begin{gathered}
E_{X A}=3.478+15.4 \varepsilon_{z z} \mathrm{eV}, \\
E_{X B}=3.484+15.4 \times 1.24 \varepsilon_{z z} \mathrm{eV} .
\end{gathered}
$$

$\varepsilon_{z z}=\left(c-c_{0}\right) / c_{0}$ denotes strain along the $c$ axis, where $c_{0}$ and $c$ are the lattice constants for the unstrained and strained crystal, respectively. Therefore, the $E_{X A}$ and $E_{X B}$ are estimated to be 3.504 and $3.517 \mathrm{eV}$. The experimental absorption peak at $3.521 \mathrm{eV}$ and the shoulder at $3.510 \mathrm{eV}$ are probably attributed to the $E_{X B}$ and $E_{X A}$, respectively, although there are small deviations ( 4 and $6 \mathrm{meV}$ ) from the theoretical values. The validity for assigning the main peak to the $\mathrm{B}$ exciton is supported from the fact that the oscillator strengths of $\mathrm{A}$ and $\mathrm{B}$ excitons are comparable, and that the band-tail absorption is preferentially superimposed on the higher energy band. It should be noted that the similar feature is also observed for a $0.38 \mu \mathrm{m}$ thick $\mathrm{GaN}$ single epilayer where the band-tailing is less dominant than the sample assessed in this study. ${ }^{15}$

Figure 2 shows the transient absorption spectra under the photoexcitation above the band gap for some optical delays. Just after the photoexcitation, the absorption decreases in the whole region because of the saturation of the absorption owing to the high carrier density (about $1.4 \times 10^{20} \mathrm{~cm}^{-3}$ ). And the absorption peak of exciton disappears because the carrier density is above Mott density $\left(10^{18} \mathrm{~cm}^{-3}\right.$ for $\mathrm{GaN}$ at 10 $\mathrm{K}){ }^{21}$ The PL lifetime at the carrier densities from $1 \times 10^{17}$ to $1.4 \times 10^{20} \mathrm{~cm}^{-3}$ is about $6-7 \mathrm{ps}$ measured by time-

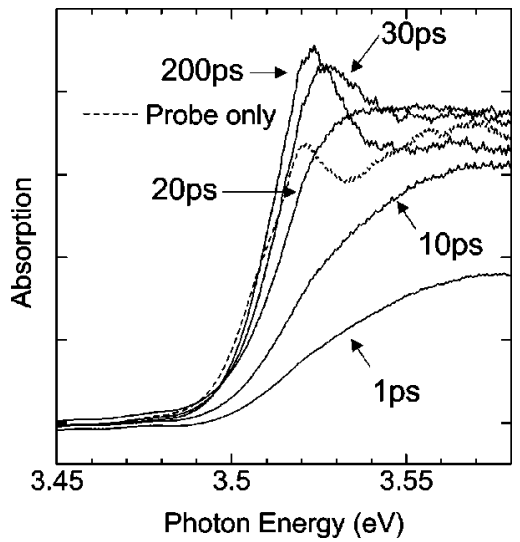

FIG. 2. Transient absorption spectra in a thin GaN epilayer at 10 $\mathrm{K}$ under the femtosecond pulse excitation $(3.646 \mathrm{eV})$. The broken curve is the absorption spectrum without a photoexcitation.

resolved-photoluminescence spectroscopy (TRPL). Assuming that the PL lifetime is $6 \mathrm{ps}(7 \mathrm{ps})$ in the time range from $0 \mathrm{ps}$ to $30 \mathrm{ps}$, the density of free carriers at $30 \mathrm{ps}$ is estimated to be less than $1 \times 10^{18} \mathrm{~cm}^{-3}\left(2 \times 10^{18} \mathrm{~cm}^{-3}\right)$ and it probably corresponds to about the same value as Mott density. Therefore, the absorption peak of excitons is restored at about $30 \mathrm{ps}$. After $30 \mathrm{ps}$, the absorption peak is shifted to the lower energy side and the absorption peak of exciton is clarified. The PL lifetime below $1 \times 10^{17} \mathrm{~cm}^{-3}$ is about 14 \pm 1 ps. So the carrier density decreased roughly less than $1 \times 10^{13} \mathrm{~cm}^{-3}$ for $200 \mathrm{ps}$. This lifetime is shorter than that (a few hundred ps) of a typical GaN epilayer. This may be because the large strain induced the formation of nonradiative recombination centers which limit the recombination channel even at low temperature. The absorption spectra from 20 to 200 ps are larger than that of without pump beam. This is because Franz-Keldysh and Stark effects due to the internal electric field suppressed the absorption without pump beam. The internal electric field strength due to piezoelectric polarization can be calculated using $\varepsilon_{z z}$, elastic constants, and piezoelectric constants. However, estimated field strength range from $0.15 \mathrm{MV} / \mathrm{cm}$ to $0.45 \mathrm{MV} / \mathrm{cm}$, mainly due to the scattering of reported values of piezoelectric constants. ${ }^{6,22,23}$ Photoinduced carriers almost eliminated Franz-Keldysh and Stark effects because of the screening of the internal electric field.

Figure 3(a) shows the spectra of the photoinduced change of optical density $(\triangle O D)$ at several optical delays. $\triangle O D$ is expressed by the following equation:

$$
\Delta O D=\log _{10}\left(\frac{T}{T+\Delta T}\right)=\Delta \alpha d \times 0.434
$$

where $T, T+\Delta T$ is the probe beam intensity in the absence and the presence of the pump beam, respectively. $\Delta \alpha$ is the photoinduced change of absorption coefficient, $d$ is the thickness of absorbing layer. Therefore, at $\Delta O D<0$ the absorption decreases due to the bleaching, and at $\triangle O D>0$ the absorption increases due to the photoinduced absorption. The bleaching was observed in the whole energy range just after 

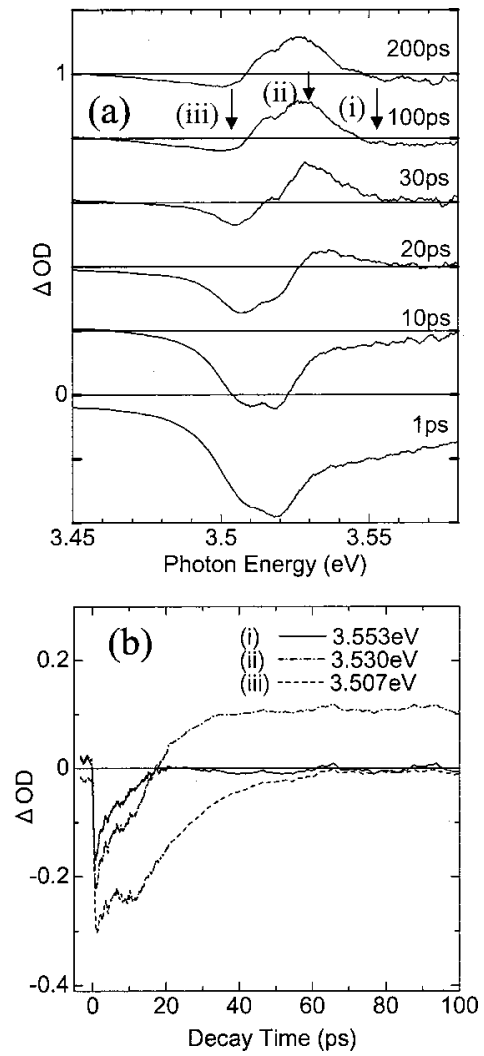

FIG. 3. (a) Differential optical density spectra taken at various time after pulsed excitation with a photon energy of $3.646 \mathrm{eV}$ (above band gap excitation) at $10 \mathrm{~K}$. (b) Temporal behavior of optical density monitored at (i) $3.553 \mathrm{eV}$, (ii) $3.530 \mathrm{eV}$, and (iii) $3.507 \mathrm{eV}$.

photoexcitation. After $20 \mathrm{ps}$, the photoinduced absorption was observed in the energy range of 3.51 to $3.55 \mathrm{eV}$. The decay curve at (i) $3.553 \mathrm{eV}$, (ii) $3.530 \mathrm{eV}$, and (iii) $3.507 \mathrm{eV}$ was shown in Fig. 3(b). The decay curves are not simple exponential curves because $\triangle O D$ is affected by the many effects such as the band filling, the Mott transition and the screening of the internal electric field. Within 20 ps after the photoexcitation, $\triangle O D$ is negative due to the saturation of the absorption owing to the high carrier density. After $20 \mathrm{ps,}$ $\triangle O D$ becomes positive at $3.530 \mathrm{eV}$. And $\triangle O D$ maintains negative at 3.553 and $3.507 \mathrm{eV}$ in the whole time region. From 40 ps to 200 ps, the decay curve keeps constant. As already mentioned, the free carrier density of 1.4 $\times 10^{20} \mathrm{~cm}^{-3}$ is decreased less than $1 \times 10^{13} \mathrm{~cm}^{-3}$ at 200 ps. Provided that the photoinduced absorption is due to the screening of the internal electric field, the carrier density of $1 \times 10^{13} \mathrm{~cm}^{-3}$ is too low to induce the screening of the internal electric field. Therefore carriers trapped at deep levels having a very long lifetime play a major role for this screening effect. Although it is difficult to estimate the exact time constant of these trapped carriers because of the limitation of time delay ( $250 \mathrm{ps}$ ) in our system, it is presumed to be more than $8 \mathrm{~ns}$ taking into account the signal to noise level within the time scale of $0-200 \mathrm{ps}$. This value is much longer than free carrier decay time estimated by TRPL. From this
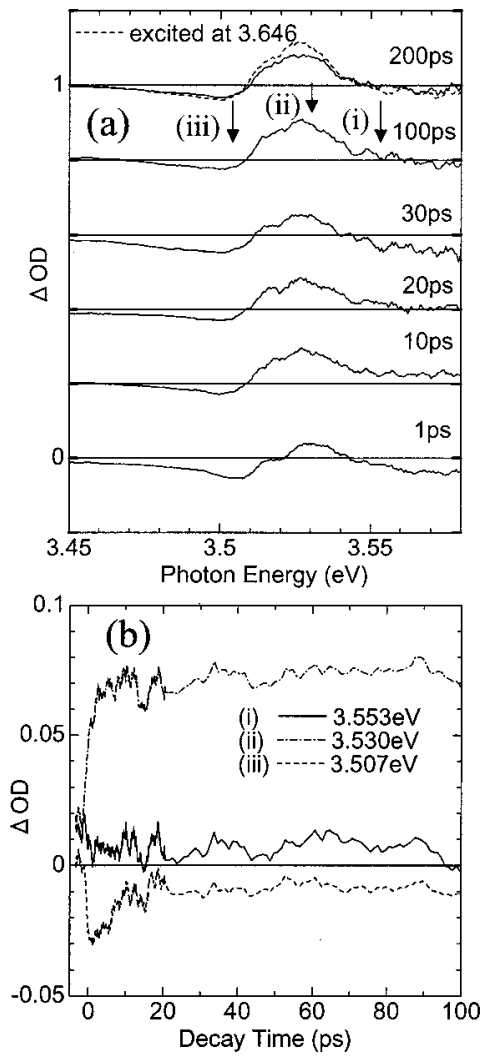

FIG. 4. (a) Differential optical density spectra taken at various time after pulsed excitation with a photon energy of $3.443 \mathrm{eV}$ (below band gap excitation) at $10 \mathrm{~K}$. The spectrum taken under above band gap excitation is shown as a broken curve for $t=200$ ps. (b) Temporal behavior of optical density monitored at (i) $3.553 \mathrm{eV}$, (ii) $3.530 \mathrm{eV}$, and (iii) $3.507 \mathrm{eV}$.

sample, PL band related to donor acceptor pair (DAP) is obtained. However, intensity of such band is one order of magnitude smaller than exciton related PL band. The zero phonon line of DAP is located at $3.304 \mathrm{eV}$ indicating that shallow levels of both donor and acceptor originate from this DAP band. Moreover, almost no deep level emission such as yellow luminescent band could not be observed. Therefore, carriers, trapped at deep levels recombine nonradiatively, although atomic or molecular origin of trapping center is unclear. It is difficult to exactly illustrate how carriers trapped at deep levels screen the electric field. However, if trapped carriers to the growth direction are not uniformly distributed, the internal electric field can be screened. We assume that after the photoinduced electron and hole are separated by electric field, each electron and hole relaxes to nonradiative recombination center at each position. Then the electron and hole trapped at deep level can screen the internal electric field, because the trapped electron and hole are distributed at different points in the growth direction.

To investigate the effect of the deep-level trapped carriers, the transient absorption spectroscopy under the photoexcitation below band gap was performed. The $\triangle O D$ spectra and decay curves under the photoexcitation at $3.443 \mathrm{eV}$ are shown in Fig. 4. The spectra under the photoexcitation below 
band gap are almost the same from 0 ps to $200 \mathrm{ps.} \mathrm{In} \mathrm{order} \mathrm{to}$ compare the effect of photoinduced excitonic absorption with that above band gap excitation, the $\triangle O D$ spectrum at 200 ps taken under $3.646 \mathrm{eV}$ is shown in Fig. 4 by the broken curve. The photoinduced absorption was also observed under the below band gap excitation although the positive signal is about $67 \%$ of that under the above band gap excitation. It is well known that ac Stark effects contribute to the photoinduced absorption under the photoexcitation below band gap. However, such effects take place within a few picoseconds in GaN. ${ }^{24}$ The observed photoinduced absorption has time constant much longer than 200 ps. Therefore, the origin of photoinduced absorption is probably ascribed to the screening of the internal electric field. The absorption coefficient at $3.443 \mathrm{eV}$ in this $\mathrm{GaN}$ epilayer with the internal electric field of 0.15 and $0.45 \mathrm{MV} / \mathrm{cm}$ was estimated to be about $0.1 \%$ and $(0.6 \%)$, respectively, of the absorption coefficient at $3.646 \mathrm{eV}$ considering Franz-Keldysh effect. ${ }^{25}$ Therefore, the carrier density just after photopumping is estimated to be $1 \times 10^{17}$ and $8 \times 10^{17} \mathrm{~cm}^{-3}$ in the electric field strength of 0.15 and $0.45 \mathrm{MV} / \mathrm{cm}$, respectively, neglecting the absorption saturation and transition between free and bound states. In the very simple model as the parallel conductive plate, the electric filed strength as large as 0.25 $\mathrm{MV} / \mathrm{cm}(2.0 \mathrm{MV} / \mathrm{cm})$ is induced if the carriers with concen- tration of $1 \times 10^{17} \mathrm{~cm}^{-3}\left(8 \times 10^{17} \mathrm{~cm}^{-3}\right)$ are spatially separated between $\mathrm{GaN}$ surface and GaN/AlGaN interface. This value is overestimated because deep level traps are distributed within $\mathrm{GaN}$ epilayer perpendicular to the growth direction. Nevertheless, it is found that carriers trapped at deep levels can screen the internal electric field even with the density of $10^{17} \mathrm{~cm}^{-3}$ order.

In conclusion, the dynamics of photoinduced absorption has been studied in a thin highly strained GaN epilayer using the transient absorption spectroscopy at low temperature. The Mott transition was observed just after the photoexcitation. Decreasing the carriers, the absorption peak of the excitons is restored. The absorption spectra became larger than that without pump beam due to the screening of the internal electric field. The time scale of this screening is more than 8 ns larger than PL lifetime. Moreover, the photoinduced absorption was also observed under the photoexcitation below band gap. The results suggest that carrier density necessary for screening piezoelectric field is in the order of $10^{17} \mathrm{~cm}^{-3}$ and the carrier trapped at deep levels contributed to such phenomena.

A part of this work was performed using the facility at the Venture Business Laboratory at Kyoto University (KU-VBL).
${ }^{1}$ S. Nakamura, M. Senoh, N. Iwasa, and S. Nagahama, Jpn. J. Appl. Phys., Part 2 34, L797 (1995).

${ }^{2}$ S. Nakamura, M. Senoh, N. Iwasa, S. Nagahama, T. Yamada, and T. Mukai, Jpn. J. Appl. Phys., Part 2 34, L1332 (1995).

${ }^{3}$ S. Nakamura, M. Senoh, S. Nagahama, N. Iwasa, T. Matsushita, H. Kiyoku, and Y. Sugimoto, Jpn. J. Appl. Phys., Part 2 35, L74 (1996).

${ }^{4}$ S. Nakamura, M. Senoh, S. Nagahama, N. Iwasa, T. Matsushita, and T. Mukai, Appl. Phys. Lett. 76, 22 (2000).

${ }^{5}$ S. Nagahama, N. Iwasa, M. Senoh, T. Matsushita, Y. Sugimoto, H. Kiyoku, T. Kozaki, M. Sano, H. Matsumura, H. Umemoto, K. Chocho, and T. Mukai, Jpn. J. Appl. Phys., Part 2 39, L647 (2000).

${ }^{6}$ T. Takeuchi, S. Sota, M. Katsuragawa, M. Komori, H. Takeuchi, H. Amano, and I. Akasaki, Jpn. J. Appl. Phys., Part 2 36, L382 (1997).

${ }^{7}$ C. Wetzel, T. Takeuchi, H. Amano, and I. Akasaki, Phys. Rev. B 62, R13 302 (2000).

${ }^{8}$ T. Kuroda, A. Tackeuchi, and T. Sota, Appl. Phys. Lett. 76, 3753 (2000).

${ }^{9}$ Y. Narukawa, Y. Kawakami, M. Funato, Sz. Fujita, Sg. Fujita, and S. Nakamura, Appl. Phys. Lett. 70, 868 (1997).

${ }^{10}$ Y. Narukawa, Y. Kawakami, Sz. Fujita, and Sg. Fujita, Phys. Rev. B 55, R1938 (1997).

${ }^{11}$ S. Chichibu, K. Wada, and S. Nakamura, Appl. Phys. Lett. 71, 2346 (1997).

${ }^{12}$ A. Satake, Y. Masumoto, T. Miyajima, T. Asatsuma, F. Nakamura, and M. Ikeda, Phys. Rev. B 57, R2041 (1998).

${ }^{13}$ S. Hess, R.A. Taylor, E.D. O'Sullivan, J.F. Ryan, N.J. Cain, V.
Roverts, and J.S. Roberts, Phys. Status Solidi B 216, 51 (1999).

${ }^{14}$ C.K. Sun, Y.-L. Huang, S. Keller, U.K. Mishra, and S.P. Denbaars, Phys. Rev. B 59, 13535 (1999).

${ }^{15}$ C.K. Choi, Y.H. Kwon, J.S. Krasinski, G.H. Park, G. Setlur, and J.J. Song Phys. Rev. B 63, 115315 (2001).

${ }^{16}$ A. Shikanai, T. Deguchi, T. Sota, T. Kuroda, A. Tackeuchi, S. Chichibu, and S. Nakamura, Appl. Phys. Lett. 76, 454 (2000).

${ }^{17}$ K. Pakula, A. Wysmolek, K.P. Korona, J.M. Baranowski, R. Stepniewski, I. Grzegory, M. Bockowski, J. Jun, S. Krukowski, M. Wroblewski, and S. Porowski, Solid State Commun. 97, 919 (1996).

${ }^{18}$ T. Detchprohm, K. Hiramatsu, K. Itoh, and I. Akasaki, Jpn. J. Appl. Phys., Part 2 31, L1454 (1992).

${ }^{19}$ A. Shikanai, T. Azuhata, T. Sota, S. Chichibu, and S. Nakamura. J. Appl. Phys. 81, 417 (1997).

${ }^{20}$ B. Gil, O. Briot, and R.L. Aulombard, Phys. Rev. B 52, R17 208 (1995).

${ }^{21}$ F. Binet, J.Y. Duboz, J. Off, and F. Scholz, Phys. Rev. B 60, 4715 (1999).

${ }^{22}$ S.F. Chichibu, T. Sota, K. Wada, O. Brandt, K.H. Ploog, S.P. Denbaars, and S. Nakamura, Phys. Status Solidi A 183, 91 (2001).

${ }^{23}$ F. Bernardini, V. Fiorentini, and D. Vanderbilt, Phys. Rev. B 56, R10 024 (1997).

${ }^{24}$ Y.C. Chang, C.K. Choi, and J.J. Song, Proc. SPIE 4280, 58 (2001).

${ }^{25}$ N. Peyghambarian, S.W. Koch, and A. Mysyrowicz, Introduction to Semiconductor Optics (Prentice Hall, New Jersey, 1993), p. 257. 